\title{
EFICIÊNCIA NA ESTIMATIVA DO PESO SECO PARA ÁRVORES INDIVIDUAIS E DEFINIÇÃO DO PONTO ÓTIMO DE AMOSTRAGEM PARA DETERMINAÇÃO DA DENSIDADE BÁSICA DE Eucalyptus camaldulensis
}

\section{EFFICIENCY IN DRY WEIGTH ESTIMATES FOR INDIVIDUAL TREES AND DEFINITION OF OPTIMUM SAMPLING POINT FOR BASIC DENSITY DETERMINATION OF Eucalyptus camaldulensis}

\author{
Edmilson Jorge Franco ${ }^{1}$ José Roberto S. Scolforo ${ }^{2}$ \\ José Márcio de Mello ${ }^{2}$ José Tarcísio de Lima²
}

\section{RESUMO}

Os objetivos deste estudo foram testar a eficiência dos modelos de dupla entrada, de simples entrada, de dupla entrada associados a relação hipsométrica e o método dos dois diâmetros; e também definir o ponto ótimo de amostragem na árvore para obter a densidade básica da madeira, a partir de uma amostra. O estudo foi conduzido na região de João Pinheiro, MG, em propriedade da Mannesman Florestal Ltda. Foram utilizadas 127 árvores cubadas rigorosamente com idades variando de 28 a 76 meses. Foram também retirados discos de metro em metro ao longo do fuste, para a determinação da densidade básica. As estimativas do peso seco total com e sem casca foram obtidas para as quatro metodologias em estudo. A comparação destas com os valores reais se deu através de um delineamento em blocos ao acaso. Foi constatado que qualquer dos procedimentos testados propiciam estimativas do peso seco semelhantes aos valores reais. No entanto, as medidas de precisão da equação de simples entrada são inferiores aos demais métodos testados. O ponto ótimo de amostragem na árvore para obter a densidade básica foi a $1 \mathrm{~m}$ de altura. Maior precisão pode ser conquistada se a amostra for tomada a 1; 3 e 6 metros de altura.

Palavras-chave: Peso seco, densidade básica, métodos de estimativa.

\begin{abstract}
The objectives of this study were to test the efficiency of models of double entrance, simple entrance, double entrance associated to hypsometric relationship, and the method of the two diameters, and also to define the optimum sampling point along the tree for obtaining the wood

1. Eng. Florestal, Técnico da Empresa Mannesman Florestal Ltda. João Pinheiro. MG.

2. Prof. do Departamento de Ciências Florestais/UFLA. CEP: 37200-000. Caixa Postal 37. Lavras. MG.
\end{abstract}


basic density from just one sample. The study was carried out in the João Pinheiro region, MG, belonging to "Mannesman Florestal" LTDA. Data from 127 cubed trees were used with ages ranging from 28 to 76 months. Cross sections cuttings at every meter of the stem were taken for basic density determination. The estimates of the total dry weigth outside and inside bark were obtained from the proposed methods. The comparison of estimated with observed values was made by randon block design. It was observed that any of tested methods resulted in dry weight estimates similar to the observed values. Meanwhile, the precision of the estimates from the simple entrance equation were inferior to those one obtained through the other tested methods. The optimum sampling point to obtain the basic density was at $1 \mathrm{~m}$ from the ground. It was also verified that a greatter precision can be obtained if the sample for basic density is taken obtained at the heights of 1,3 and 6 meters from the ground.

Key words: Dry weight, basic density, methods of estimation.

\section{INTRODUÇÃO}

Quando o objetivo do empreendimento florestal é produzir madeira para energia é mais interessante quantificar o estoque de biomassa, do que o estoque volumétrico em um povoamento florestal. A título de ilustração pode-se considerar um plantio de dois povoamentos, um de Eucalyptus camaldulensis (1) e outro de Eucalyptus urophylla (2) de mesma idade, em um mesmo local e sujeitos ao mesmo manejo. As espécies 1 e 2 produzem aos 7 anos respectivamente 150 e 160 $\mathrm{m}^{3}$ de madeira por hectare. Do ponto de vista volumétrico a espécie (2) é mais viável economicamente. Assumindo que a densidade básica da espécie (1) seja $0,59 \mathrm{~g} / \mathrm{cm}^{3}$ e da espécie (2) $0,50 \mathrm{~g} / \mathrm{cm}^{3}$, e se o produto final for energia, a primeira pode gerar $88,5 \mathrm{ton} / \mathrm{m}^{3}$ de madeira seca e a segunda 80,0 ton $/ \mathrm{m}^{3}$. Assim, após considerar o produto final, a espécie (1) é mais viável economicamente.

Corroborando com o ponto de vista apresentado anteriormente, Finke Herrera (1989) considera o peso seco, como sendo uma unidade de medida da produção e produtividade florestal a ser transformada em carvão, celulose e fibras para chapa, mais concreta que a quantificação do volume.

A estimativa do peso seco é obtida pelo produto do volume real e a densidade básica da própria árvore que compõe a amostra das árvores cubadas rigorosamente. A determinação da densidade da madeira pode ser feita através do método de imersão; método de saturação; método da balança, e outros, Vital (1984).

A densidade básica da madeira é uma propriedade física muito influenciada por vários fatores: componentes anatômicos e químicos da madeira, idade e condições edafoclimáticas. A densidade básica varia ao longo do fuste e no sentido radial. Esta comprovação já foi relatada por vários autores, podendo-se citar dentre outros, Panshin e Zeuw (1970), Shimoyama (1990) e Silva (1991).

Foelkel, Mora e Menorhelli (1990) caracterizaram a densidade básica da madeira como um 
dos principais índice para avaliar a qualidade da madeira para diversos fins. Trugilho, Silva, Frazão e Regazzi (1991) fizeram a mesma consideração sobre a importância da densidade básica quando a madeira é utilizada para fins energéticos.

Este trabalho teve como objetivos testar a eficiência de modelos de dupla entrada, de simples entrada, de dupla entrada associados à relação hipsométrica e o método dos dois diâmetros; e também definir o ponto ótimo de amostragem na árvore, para obter a densidade básica da madeira, a partir de uma amostra.

\section{MATERIAL E MÉTODOS}

\section{Dados básicos}

O estudo foi realizado em um maciço florestal de Eucalyptus camaldulensis localizado na Fazenda Patagônia no Município de João Pinheiro (MG), da Mannesmann Florestal Ltda.

Para quantificação da variável peso seco, foram utilizadas 127 árvores distribuídas em diferentes classes de diâmetro e altura. $\mathrm{O}$ espaçamento por ocasião do plantio foi de 3 x $2,5 \mathrm{~m}$ e as idades amostradas variaram de 28 a 76 meses. A distribuição das árvores amostradas por classe de diâmetro e altura é apresentada na Tabela 1.

TABELA 1: Distribuição de freqüência das árvores amostradas na cubagem rigorosa por classe de DAP e classe de altura.

\begin{tabular}{|c|c|c|c|c|c|c|c|c|}
\hline \multirow{2}{*}{$\begin{array}{c}\text { Classes de DAP } \\
(\mathrm{cm})\end{array}$} & \multicolumn{8}{|c|}{ Valor central das classes de altura total (m) } \\
\hline & 9 & 11 & 13 & 15 & 17 & 19 & 21 & Total \\
\hline $5,0-7,5$ & 5 & 8 & & & & & & 13 \\
\hline $7,5-10,0$ & 2 & 20 & 11 & 2 & & & & 35 \\
\hline $10,0-12,5$ & & 1 & 2 & 7 & 16 & 2 & & 28 \\
\hline $12,5-15,0$ & & & & 2 & 19 & 14 & & 35 \\
\hline $15,0-17,5$ & & & & & & 9 & 4 & 13 \\
\hline $17,5-20,0$ & & & & & 1 & 1 & 1 & 3 \\
\hline Total & 7 & 29 & 13 & 11 & 36 & 26 & 5 & 127 \\
\hline
\end{tabular}

Para fins de determinação volumétrica foi medido o diâmetro de 0,30 em $0,30 \mathrm{~m}$ desde a base até $3 \mathrm{~m}$ de altura. Após esta altura os diâmetros foram coletados de metro em metro, utilizando-se de suta com graduação de $0,1 \mathrm{~cm}$. O volume foi calculado pela fórmula de Smalian. De forma complementar foi medida a espessura da casca, o que possibilitou obter o volume total sem casca.

A densidade básica da madeira foi obtida de discos retirados de 1 em 1 metro, da base da árvore até $3 \mathrm{~cm}$ de diâmetro com casca, tendo os discos espessura de 2,5 cm.

O volume verde de cada disco foi obtido pelo método de imersão como descrito por Vital (1984), e utilizado também por Brasil, Veiga e Timoni (1994). Foi obtido também o peso seco em estufa, a uma temperatura de $103 \pm 2^{\circ} \mathrm{C}$, por um período de 48 horas, até um peso seco constante 
dos discos. A densidade básica média ponderada de cada árvore, foi obtida pela fórmula proposta por Barrichelo, Brito e Migliorini (1982). Do produto da densidade básica e o volume real da árvore obteve-se o peso seco.

$$
D B_{p}=\left\{\sum_{i=1}^{n}\left[\left(D_{i}^{2}+D_{i+1}^{2}\right) \cdot\left(\mathrm{DB}_{\mathrm{i}}+D B_{i+1}\right)\right] /\left[\sum_{i=2}^{n-1} 2 D_{i}+\left(D_{1}+D_{n}\right)\right]\right\} \cdot 0,5
$$

em que:

$\mathrm{DB}_{\mathrm{p}}=$ densidade básica média ponderada de cada árvore

$\mathrm{DB}_{\mathrm{i}}=$ densidade do iésimo disco

$\mathrm{D}_{\mathrm{i}}=$ diâmetro do iésimo disco

$\mathrm{D}_{1}, \mathrm{D}_{\mathrm{n}}=$ diâmetro das extremidades da porção considerada na árvore

$\mathrm{n}$ = número de discos coletados por árvore

\section{Modelos para expressar biomassa (peso seco)}

Para estimar o peso seco, foram utilizados modelos de simples entrada, dupla entrada e o método dos dois diâmetros, podendo ter como variável dependente o peso seco total com e sem casca. Os modelos de simples e dupla entrada podem ser visto em Scolforo (1993) e Franco (1996).

\section{Modelo de dupla entrada com relação hipsométrica}

Utilizou-se a relação hipsométrica associada as equações de biomassa de dupla entrada. As relações hipsométricas foram ajustadas, controlando-se o efeito idade, sítio e espaçamento. Os modelos testados são apresentados na Tabela 2.

TABELA 2: Modelos hipsométricos típicos.

\begin{tabular}{l|c}
\hline Autores/modelo & \multicolumn{1}{c}{ Modelos } \\
\hline Trorey & $\mathrm{H}=\beta_{0}+\beta_{1} \mathrm{D}+\beta_{2} \mathrm{D}^{2}+\mathrm{e}_{\mathrm{i}}$ \\
Stofel & $\mathrm{Log} \mathrm{H}=\beta_{0}+\beta_{1} \log \mathrm{D}+\log \mathrm{e}_{\mathrm{i}}$ \\
Petterson & $\mathrm{H}=1 /\left(\beta_{0}+\beta_{1} / \mathrm{D}\right)^{1 / 3}+\mathrm{e}_{\mathrm{i}}$ \\
Prodan & $\mathrm{H}=\left(\mathrm{D}^{2}\right) / \beta_{0}+\beta_{1} \mathrm{D}+\beta_{2} \mathrm{D}^{2}+\mathrm{e}_{\mathrm{i}}$ \\
\hline
\end{tabular}

\section{Métodos de dois diâmetros}

A questão central neste método é que a biomassa de qualquer porção da árvore é altamente correlacionada com a biomassa total ou mesmo com qualquer biomassa comercial. Desta maneira o interesse é saber qual porção da árvore de Eucalyptus camaldulensis é mais correlacionada com a biomassa total com e sem casca.

Buscando responder a esta pergunta, a cubagem rigorosa foi efetuada de $0,30 \mathrm{~m}$ em $0,30 \mathrm{~m}$ desde a base da árvore até a altura de 3,0 m. Foi calculado o volume das várias seções, tendo sempre 
como uma das extremidades o diâmetro a 1,30 m (DAP). Obteve-se também a biomassa de 0,00 $1,30 \mathrm{~m} ; 0,30$ - 1,30 m; 0,60 - 1,30 m; 0,90 - 1,30 m; 1,20 - 1,30 m; 1,30 - 1,50 m; 1,30 - 1,80 m; 1,30 - 2,10 m; 1,30 - 2,40 m; 1,30 - 2,70 m; 1,30 - 3,0 m. O peso seco de cada torete (Vi), foi correlacionado ao peso seco total da árvore, visando detectar qual seção, apresentou maior correlação com o peso seco total da árvore com e sem casca. O desenvolvimento do método é descrito a seguir:

Utilizando-se a equação da reta estabelece a relação entre o volume do torete e o volume da árvore:

$$
P S=\beta_{0}+\beta_{1} \cdot\left(P S_{i}\right)
$$

em que:

$\mathrm{PS}=$ peso seco total da árvore

$\beta_{\mathrm{i}}=$ parâmetros a serem estimados

$\mathrm{PS}_{\mathrm{i}}=$ peso seco entre o DAP ou D e o diâmetro (Di) a uma iésima altura (Hi)

Como o peso seco de qualquer pequena seção pode ser obtido através da fórmula de Smalian tem-se:

$$
P S_{i}=\left\{\left[\frac{\left(\pi \cdot D^{2} / 40000\right)+\left(\pi \cdot \mathrm{D}_{\mathrm{i}}^{2} / 40000\right)}{2}\right] \cdot L\right\} \cdot D B_{p}
$$

Então substituindo (2) em (1), a equação ajustada pode assumir a forma:

$$
\begin{aligned}
& P S=\beta_{0}+\left\{\beta_{1} \cdot\left[\frac{\left(\pi \cdot D^{2} / 40000\right)+\left(\pi \cdot \mathrm{D}_{\mathrm{i}}^{2} / 40000\right)}{2}\right] \cdot L\right\} \cdot D B_{p} \\
& P S=\beta_{0}+\left[\beta_{1} \cdot\left(\frac{\pi}{80000}\right) \cdot L \cdot\left(\mathrm{D}^{2}+D_{i}^{2}\right)\right] \cdot D B_{p}
\end{aligned}
$$

Sendo $K=\left(\frac{\pi}{80000}\right) \cdot L \cdot \mathrm{DB}_{\mathrm{p}}$

$$
P S=\beta_{0}+\beta_{1} \cdot K \cdot\left(D^{2}+D_{i}^{2}\right)
$$

em que:

$\mathrm{D}=\mathrm{DAP}=$ diâmetro a $1,30 \mathrm{~m}$ de altura

$\mathrm{D}_{\mathrm{i}}=$ diâmetro a iésima altura

$\mathrm{L}=$ comprimento da seção em relação ao diâmetro tomado a 1,30 m

$\beta_{\mathrm{i}}=$ parâmetro da equação

$\mathrm{DBp}=$ densidade básica ponderada da árvore 
A expressão 3 é a forma final de trabalho. A identificação de qual conjunto de diâmetro levou a uma biomassa de torete que mais se correlacionou a biomassa total da árvore foi através da regressão linear simples. Identificado o par de diâmetro, pode-se dar maior praticidade ao método através da eliminação da medida do outro diâmetro que não o DAP, a nível das parcelas do inventário. Para tal utilizando-se os dados da cubagem rigorosa pode-se estabelecer uma relação linear entre o diâmetro a qualquer altura e o DAP ou D. Está apresentado na Tabela 3 os modelos lineares que foram testados. Para efeito ilustrativo, foi utilizado a equação da linha reta.

$$
D_{i}=\beta_{2}+\beta_{3} . \mathrm{D}
$$

Substituindo então (4) em (3) tem-se:

$$
P S=\beta_{0}+\beta_{1} \cdot K \cdot\left[\mathrm{D}^{2}+\left(\beta_{2}+\beta_{3} \cdot D\right)^{2}\right]
$$

TABELA 3: Modelos matemáticos para estimativa do diâmetro a diferentes alturas.

\begin{tabular}{ll}
\hline Nome do modelo & \multicolumn{1}{c}{ Modelos } \\
\hline Reta & $D_{i}=\beta_{0}+\beta_{0} \mathrm{D}$ \\
Hipérbole & $\mathrm{D}_{\mathrm{i}}=\beta_{0}+\beta_{0}(1 / \mathrm{D})$ \\
Reta (logaritimizada) & $\log \mathrm{D}_{\mathrm{i}}=\beta_{0}+\beta_{1} \operatorname{LogD}$ \\
\hline
\end{tabular}

em que:

Di, $\mathrm{D}, \log =$ já foram definidos anteriormente

\section{Ponto ótimo para obter a densidade básica da madeira}

Para identificar a altura na árvore cuja amostra represente a densidade média da árvore, foi utilizada a técnica de regressão, através do ajuste dos modelos lineares listados na Tabela 4. Nestes a variável dependente foi a densidade básica ponderada da árvore e as variáveis independentes foram as densidades a $0,0 \mathrm{~m} ; 1,0 \mathrm{~m} ; 1,30 \mathrm{~m} ; 2,0 \mathrm{~m} ; 3,0 \mathrm{~m} ; 4,0 \mathrm{~m} ; 5,0 \mathrm{~m} ; 6,0 \mathrm{~m}$; e a 1,3 e 6 metros.

A relação que apresentar maior correlação, melhor distribuição dos resíduos e menor erro padrão residual é que definirá o ponto ótimo onde deverá ser coletado o material lenhoso para fins de estimativa da densidade básica da árvore.

O estudo foi realizado a nível de idade e genericamente através do agrupamento das árvores cubadas nas diferentes idades.

em que:

$\mathrm{DB}_{\mathrm{p}}=$ é a densidade básica ponderada da árvore

$\mathrm{DB}_{\mathrm{i}}=$ é a densidade básica na iésima altura

$\beta_{\mathrm{i}}, \mathrm{e}_{\mathrm{i}}=\mathrm{já}$ foram definidos anteriormente 
TABELA 4: Modelos ajustados para identificar ponto de amostragem para densidade básica da madeira.

\begin{tabular}{l|l}
\hline Característica de interesse & \multicolumn{1}{c}{ Modelos } \\
\hline & $\mathrm{DB}_{\mathrm{p}}=\beta_{0}+\beta_{1} \mathrm{DB}_{\text {base }}+\mathrm{e}_{\mathrm{i}}$ \\
& $\mathrm{DB}_{\mathrm{p}}=\beta_{0}+\beta_{1} \mathrm{DB}_{1 \mathrm{~m}}+\mathrm{e}_{\mathrm{i}}$ \\
& $\mathrm{DB}_{\mathrm{p}}=\beta_{0}+\beta_{1} \mathrm{DB}_{2 \mathrm{~m}}+\mathrm{e}_{\mathrm{i}}$ \\
Densidade Básica & $\mathrm{DB}{ }_{\mathrm{p}}=\beta_{0}+\beta_{1} \mathrm{DB}_{\mathrm{DAP}}+\mathrm{e}_{\mathrm{i}}$ \\
da madeira $\left(\mathrm{g} / \mathrm{cm}^{3}\right)$ & $\mathrm{DB}=\beta_{0}+\beta_{1} \mathrm{DB}_{3 \mathrm{~m}}+\mathrm{e}_{\mathrm{i}}$ \\
& $\mathrm{DB}=\beta_{0}+\beta_{1} \mathrm{DB}_{4 \mathrm{~m}}+\mathrm{e}_{\mathrm{i}}$ \\
& $\mathrm{DB}_{\mathrm{p}}=\beta_{0}+\beta_{1} \mathrm{DB}_{5 \mathrm{~m}}+\mathrm{e}_{\mathrm{i}}$ \\
& $\mathrm{DB}_{\mathrm{p}}=\beta_{0}+\beta_{1} \mathrm{DB}_{6 \mathrm{~m}}+\mathrm{e}_{\mathrm{i}}$ \\
& $\mathrm{DB}_{\mathrm{p}}=\beta_{0}+\beta_{1} \mathrm{DB}_{1 \mathrm{~m}}+\beta_{2} / \mathrm{DB}_{3 \mathrm{~m}}+\beta_{3} \mathrm{DB}_{6 \mathrm{~m}}+\mathrm{e}_{\mathrm{i}}$ \\
\hline
\end{tabular}

\section{Critérios para seleção dos modelos}

A seleção da equação mais eficiente na estimativa do peso seco baseou-se no:

- Coeficiente de determinação corrigido $\left(\mathrm{R}^{2}\right)$ mais próximo de 1 ;

- Erro padrão residual em percentagem (Syx \%) na escala da variável original mais próximo de zero; e

- Distribuição gráfica dos resíduos sem tendências.

\section{Eficiência dos procedimentos}

Utilizou-se de um delineamento experimental de blocos ao acaso (DBC) em que as classes de diâmetro constituíram os blocos. A média da biomassa real de cada árvore, deu origem a testemunha 1 (T1); a média da biomassa estimada através da equação de simples entrada, o tratamento 2 (T2); a média da biomassa estimada através da equação de dupla entrada, o tratamento 3 (T3); a média da biomassa estimada através da equação de dupla entrada associada a relação hipsométrica, o tratamento 4 (T4); e a média da biomassa estimada através do método dos dois diâmetros, o tratamento 5 (T5).

Utilizou-se para este experimento 127 árvores que compuseram a amostra da cubagem rigorosa. Estas foram agrupadas em classes diamétrica para melhor controlar a variabilidade. Assim foram definidos 7 blocos. O primeiro compreendeu as árvores com diâmetro entre 3,1 e 6,0 cm; o segundo compreendeu as árvores com diâmetro entre 6,1 e 9,0 cm; e sucessivamente até totalizar 7 classes diamétricas.

Se for detectada diferença significativa entre os tratamentos será executado o teste de média Scott-Knott, conforme Scott-Knott (1974), considerando nível de significância $\alpha=0,05$. 


\section{RESULTADOS E DISCUSSÃO}

\section{Estimativa do peso seco através da equação de simples entrada}

$\mathrm{Na}$ Tabela 5, são apresentadas as equações selecionadas, para estimar o peso seco total com e sem casca, assim como suas medidas de precisão, coeficiente de determinação $\left(\mathrm{R}^{2}\right)$ e erro padrão residual (Syx). Na Figura 1, são apresentadas as distribuições gráficas dos resíduos para as equações selecionadas.

TABELA 5: Parâmetros estimados e medidas de precisão das equações para estimar peso seco.

\begin{tabular}{l|c|c|c|c}
\hline Peso Seco $(\mathrm{kg})$ & Equações & $\mathrm{R}^{2}$ & Syx & Syx (\%) \\
\hline Total c/c & LogPStc/c $=-9,609445+2.639424 \operatorname{LogD}$ & 0,972 & 0,00676 & 14,20 \\
Total s/c & LogPSts/c $=-9,947055+2.696071 \operatorname{LogD}$ & 0,962 & 0,00650 & 16,47 \\
\hline
\end{tabular}

em que:

$\mathrm{D}$ ou DAP = diâmetro tomado a $1,30 \mathrm{~m}$

Log = logarítimo natural

$\mathrm{PStc} / \mathrm{c}=$ peso seco total com casca

PSts/c $=$ peso seco total sem casca

O modelo que melhor se ajustou aos 4 conjuntos de dados foi o logarítmico, e conforme pode-se observar pelas medidas de precisão, apresentaram valor mínimo de coeficiente de determinação $\left(\mathrm{R}^{2}\right) 96 \%$ e valor máximo de erro padrão da estimativa de 16,47\%. Conforme observado na Figura 1 para as 2 situações de estudo a estimativa da biomassa é obtida sem tendenciosidade para qualquer classe de diâmetro.

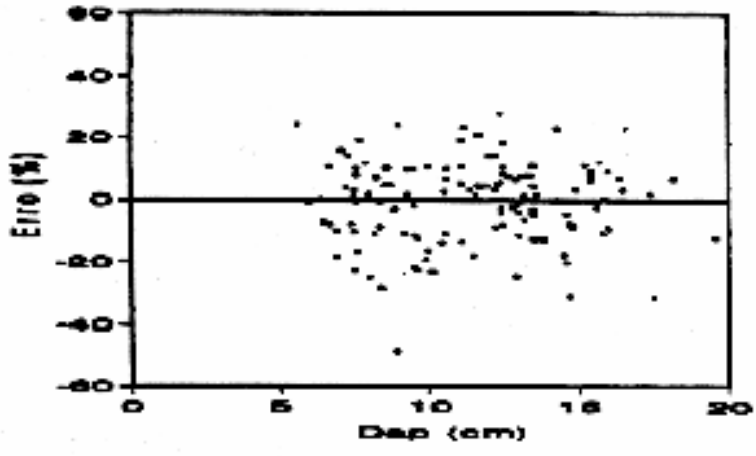

(a)

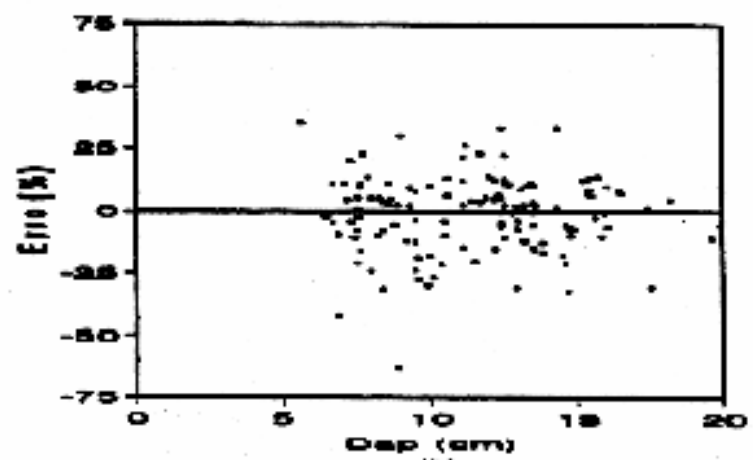

(b)

FIGURA 1: Distribuição gráfica dos resíduos para a equação de peso seco total com casca (a), peso seco total sem casca (b).

\section{Estimativa do peso seco através da equação de dupla entrada}

$\mathrm{Na}$ Tabela 6, são apresentadas as equações selecionadas para estimar o peso seco total com e 
sem casca, assim como suas medidas de precisão.

TABELA 6: Parâmetros estimados e medidas de precisão das equações para estimar peso seco.

\begin{tabular}{c|c|c|c|c}
\hline $\begin{array}{c}\text { Peso Seco } \\
(\mathrm{kg})\end{array}$ & Equações & $\mathrm{R}^{2}$ & Syx & $\begin{array}{c}\text { Syx } \\
(\%)\end{array}$ \\
\hline Total c/c & Pstc/c $=0,002222-0,000062 \mathrm{D}^{2}+0,00002 \mathrm{D}^{2} \cdot \mathrm{H}+0,000001405 \mathrm{D} \cdot \mathrm{H}^{2}$ & 0,972 & 0,00547 & 11,5 \\
Total s/c & Psts $/ \mathrm{c}=0,003170-0,000128 \mathrm{D}^{2}+0,00002 \mathrm{D}^{2} \cdot \mathrm{H}+0,000001820 \mathrm{D} \cdot \mathrm{H}^{2}$ & 0,967 & 0,00547 & 12,8 \\
\hline
\end{tabular}

em que:

$\mathrm{H}=$ altura total da árvore

Pstc/c, Psts/c = já foram definidos anteriormente

O modelo que melhor se ajustou as duas situações sob estudo foi construído através do método "Stepwise". Também Oliveira, Lima e Rosado (1991) e Scolforo, Lima e Silva (1993) construiram modelos para representar a variável peso seco, já que não obtiveram ajustes satisfatórios para o elenco de modelos consultados na literatura florestal. Comparativamente com as equações de simples entrada, houve uma queda média de $3 \%$ na estimativa do erro padrão residual, quando utilizou-se as equações de dupla entrada. Desta forma, mais uma vez ficou claro que a altura é uma variável que tem uma participação importante no modelo. Uma análise mais detalhada sobre a vantagem ou não de se utilizar esta variável, no modelo, será apresentada posteriormente.

Na Figura 2, pode-se observar que a distribuição gráfica dos resíduos para estimativa da biomassa, para árvores com quaisquer valores de diâmetro, não foi tendenciosa para o peso seco total com casca e superestimou o peso seco sem casca.

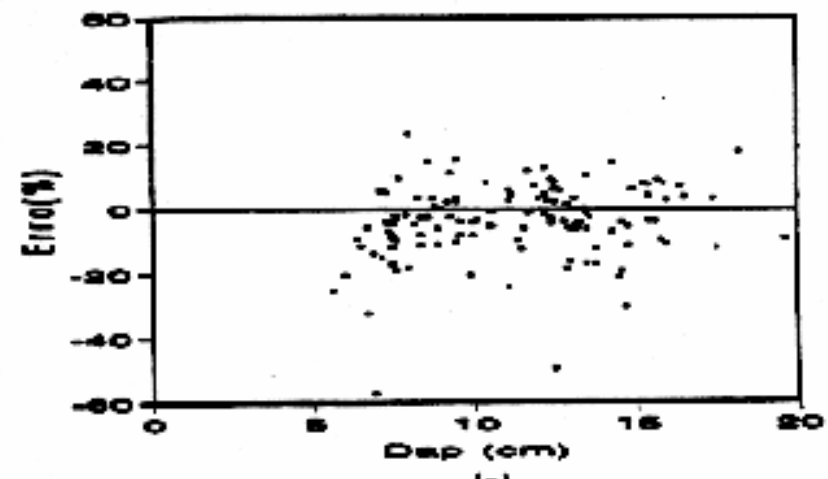

(a)

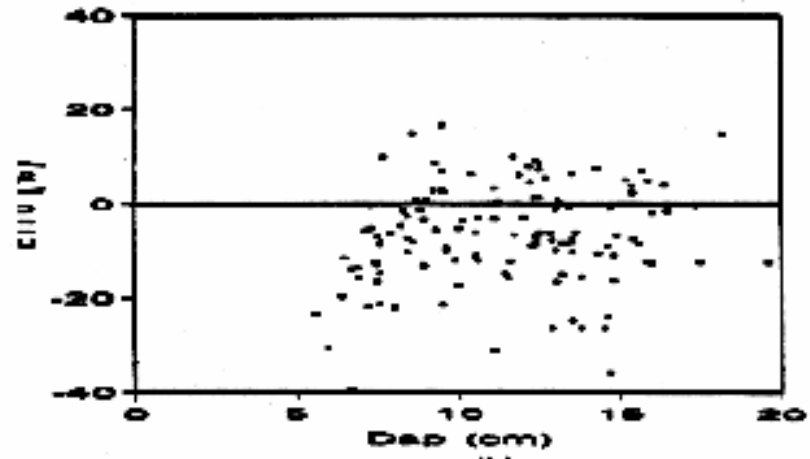

(b)

FIGURA 2: Distribuição gráfica dos resíduos para a equação de peso seco total com casca (a), peso seco total sem casca (b).

\section{Relação hipsométrica associada a equação de biomassa de dupla entrada}

Na Tabela 7 são apresentadas as equações selecionadas, a nível de talhão.

Estas equações em conexão com as equações de biomassa de dupla entrada, possibilitarão a 
obtenção de estimativas da biomassa a custos sensivelmente mais baixos, já que neste caso somente parte das árvores tem suas alturas mensuradas na parcela do inventário florestal.

TABELA 7: Equações hipsométricas e suas medidas de precisão.

\begin{tabular}{|c|c|c|c|c|}
\hline Talhão & Equações selecionadas & $\mathrm{R}^{2}$ & Syx & Syx \% \\
\hline 1 & $\mathrm{H}=\left(\left(\mathrm{D}^{2}\right) /\left(-6,11428+1,974551 \mathrm{D}-0,056181 \mathrm{D}^{2}\right)\right)+1,3$ & 0,64 & 0,90563 & 7,4 \\
\hline 2 & $H=\left(\left(D^{2}\right) /\left(-0,72603+0,58003 D+0,019199 D^{2}\right)\right)+1,3$ & 0,78 & 1,00506 & 5,8 \\
\hline 3 & $H=\left(\left(D^{2}\right) /\left(0,21891+0,38239 D+0,027555 D^{2}\right)\right)+1,3$ & 0,79 & 1,04479 & 6,0 \\
\hline 4 & $\mathrm{H}=\left(\left(\mathrm{D}^{2}\right) /\left(-5,57364+0,95195 \mathrm{D}+0,011697 \mathrm{D}^{2}\right)\right)+1,3$ & 0,88 & 0,83868 & 4,1 \\
\hline
\end{tabular}

\section{Estimativa do peso seco através do método dos dois diâmetros}

A partir do estudo da correlação, do erro padrão da estimativa e do erro padrão da estimativa em percentagem, verificou-se que o torete de 1,30 m até 3,0 m, foi o que melhor se correlacionou com a biomassa total da árvore. Este mesmo torete foi considerado para fazer estimativa da variável peso seco total sem casca.

Na Tabela 8, estão apresentadas as equações selecionadas, assim como suas medidas de precisão. Na Figura 3 são apresentadas as distribuições de resíduos para as equações selecionadas.

TABELA 8: Parâmetros estimados e medidas de precisão das equações para estimar peso seco.

\begin{tabular}{c|c|c|c|c}
\hline $\begin{array}{c}\text { Peso Seco } \\
(\mathrm{kg})\end{array}$ & Equações & $\mathrm{R}^{2}$ & Syx & $\begin{array}{c}\text { Syx } \\
(\%)\end{array}$ \\
\hline $\begin{array}{c}\text { Total c/c } \\
\text { Total stc/c }=-0,021181+3,970161\left[\mathrm{k} \cdot\left(\mathrm{D}^{2}+\mathrm{D}^{2}{ }_{3 \mathrm{~m}}\right)\right]+0,000059 /\left[\mathrm{k}\left(\mathrm{D}^{2}+\mathrm{D}^{2}{ }_{3 \mathrm{~m}}\right)\right]\end{array}$ & 0,971 & 0,00552 & 11,6 \\
\hline
\end{tabular}

em que:

$D_{3 m}=-0,81459+0,92588 . D$

$\mathrm{k}=(3,1416 / 80000) \cdot 1,7$

Log, Pstc/c, Psts/c = já foram definidos anteriormente

Pode-se observar na Tabela 8 e Figura 3 que o desempenho do método dos dois diâmetros é satisfatório, semelhante ao das equações de dupla entrada. Este fato pode levar o manejador florestal a fazer uso de uma metodologia que implicará em grande economia nos levantamentos florestais.

Para evitar a medida do diâmetro a 3 metros de altura da árvore pode-se fazer uso da relação $\mathrm{D}_{3 \mathrm{~m}}=-0,81459+0,925881 \mathrm{D}$, com $\left(\mathrm{R}^{2}=0,99\right) ;($ Syx $\%=8,7)$, definida para a população em questão. 

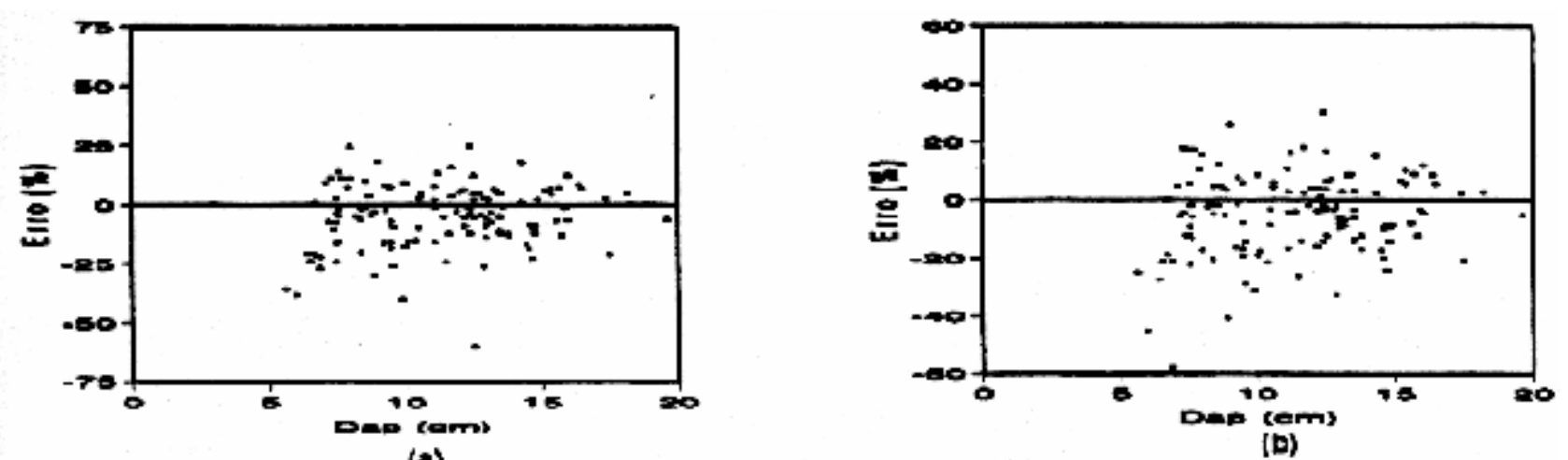

FIGURA 3: Distribuição gráfica dos resíduos para a equação de peso seco total com casca (a), peso seco total sem casca (b).

\section{Eficiência dos métodos sob estudo}

Nas Tabelas 9 e 10 estão apresentados os valores médios do peso seco total com e sem casca para cada uma das 7 classes de diâmetros, cujas árvores foram cubadas rigorosamente e para os cinco tratamentos sob estudo. Em todas as situações foi efetuado o teste de normalidade de Bartlett, conforme Gomes (1984), constatando-se normalidade dos dados ao nível de significância $\alpha$ $=0,05$.

TABELA 9: Pesos secos médios total real com casca e os estimados pelos quatro métodos sob estudo (experimento 1).

\begin{tabular}{c|c|c|c|c|c}
\hline \multirow{2}{*}{ Blocos } & \multicolumn{5}{|c}{ Tratamentos } \\
\cline { 2 - 6 } & 1 & 2 & 3 & 4 & 5 \\
\hline 1 & 0,009220 & 0,009326 & 0,010075 & 0,010175 & 0,010475 \\
3 & 0,015820 & 0,015970 & 0,015583 & 0,015381 & 0,015245 \\
4 & 0,026632 & 0,027706 & 0,026078 & 0,027707 & 0,027064 \\
5 & 0,051143 & 0,048286 & 0,049769 & 0,049562 & 0,049512 \\
6 & 0,067482 & 0,069426 & 0,070223 & 0,070442 & 0,070461 \\
7 & 0,102360 & 0,097542 & 0,098961 & 0,097279 & 0,096290 \\
& 0,133335 & 0,142285 & 0,130678 & 0,136234 & 0,133783 \\
\hline
\end{tabular}

em que:

$\mathrm{T} 1=$ Testemunha (peso seco real das árvores cubadas)

$\mathrm{T} 2$ = Peso seco estimado pela equação de simples entrada

T3 = Peso seco estimado pela equação de dupla entrada

T4 = Peso seco estimado pela equação de dupla entrada associada à relação hipsométrica típica

T5 = Peso seco estimado pelo método dos dois diâmetros 
TABELA 10: Pesos secos médios total real sem casca, e os estimados pelos métodos sob estudo (experimento 2).

\begin{tabular}{c|c|c|c|c|c}
\hline \multirow{2}{*}{ Blocos } & \multicolumn{5}{|c}{ Tratamentos } \\
\cline { 2 - 6 } & 1 & 2 & 3 & 4 & 5 \\
\hline 1 & 0,006564 & 0,006826 & 0,007576 & 0,007485 & 0,007962 \\
2 & 0,012041 & 0,012042 & 0,011802 & 0,011515 & 0,011476 \\
3 & 0,020648 & 0,021528 & 0,020222 & 0,021712 & 0,021171 \\
4 & 0,041635 & 0,038687 & 0,040431 & 0,040088 & 0,040182 \\
5 & 0,054913 & 0,056752 & 0,057707 & 0,057981 & 0,057926 \\
6 & 0,085235 & 0,081221 & 0,082367 & 0,080438 & 0,079795 \\
7 & 0,110961 & 0,121020 & 0,108527 & 0,113439 & 0,111513 \\
\hline
\end{tabular}

em que:

T1, T2, T3, T4 e T5 = já definidos anteriormente.

A partir dos valores apresentados nestas tabelas pode-se comparar através do delineamento em blocos casualizados (DBC), o peso seco médio paramétrico, com os pesos secos estimados para as duas situações sob estudo.

Os resultados da análise de variância para peso seco total com e sem casca são apresentados nas Tabelas 11 e 12 respectivamente. Os resultados mostram que não existe diferença significativa para estimar peso seco, através de qualquer um dos métodos testados. As estimativas do peso seco poderão então ser obtidas a custos reduzidos, mesmo se maior precisão nas estimativas for desejada, já que o método das duas toras apresenta equações com medidas de precisão similares às equações de dupla entrada.

TABELA 11: Análise de variância para peso seco total com casca.

\begin{tabular}{l|r|c|c|c|c}
\hline \multicolumn{1}{c|}{$\begin{array}{c}\text { Fontes de } \\
\text { Variação }\end{array}$} & G.L. & Soma de Quadrado & Quadrado Médio & F & Signif. \\
\hline Tratamento & 4 & 0,0000073575 & 0,0000018394 & 0,415 & ns \\
Bloco & 6 & 0,0644376200 & 0,0107396000 & 2425,144 & 0,000000 \\
Resíduo & 24 & 0,0001062825 & 0,0000044284 & & \\
\hline Total & 34 & & & \\
\hline
\end{tabular}

TABELA 12: Análise de variância para peso seco total sem casca.

\begin{tabular}{l|r|c|c|c|c}
\hline \multicolumn{1}{c}{$\begin{array}{c}\text { Fontes de } \\
\text { Variação }\end{array}$} & G.L. & Soma de Quadrado & Quadrado Médio & F & Signif. \\
\hline Tratamento & 4 & 0,00000953 & 0,00000238 & 0,756 & $\mathrm{~ns}$ \\
Bloco & 6 & 0,04725524 & 0,00787587 & 2497,536 & 0,00000 \\
Resíduo & 24 & 0,00007568 & 0,00000315 & & \\
\hline Total & 34 & & \\
\hline
\end{tabular}




\section{Definição do ponto ótimo de amostragem na árvore para obtenção da densidade básica}

Observou-se que a maior correlação de uma variável em relação a biomassa total foi quando o material lenhoso foi coletado a 1 metro de altura na árvore. Este fato é confirmado ao observar a regressão linear apresentada na Tabela 13, evidenciando um coeficiente de determinação de $91 \%$. A distribuição de resíduos apresentada na Figura 4(a), corrobora com a afirmação anterior. Somente para as árvores com diâmetro próximo a $20 \mathrm{~cm}$ houve tendência de superestimativa. Para as demais alturas testadas o comportamento foi similar ao mostrado na Figura 4 (b), ou seja, tendenciosidade em todas as classes de diâmetro.

TABELA 13: Equações para estimativa da densidade básica da madeira de Eucalyptus camaldulensis em relação à posição de amostragem na árvore.

\begin{tabular}{c|c|c|c}
\hline $\begin{array}{c}\text { Característica de } \\
\text { interesse }\end{array}$ & Equações & $\mathrm{R}^{2}(\%)$ & Syx $(\%)$ \\
\hline & $\mathrm{DB}_{\mathrm{p}}=0,16405+0,71623 * \mathrm{DB}$ & 0,79 & 2,79 \\
& $\mathrm{DB}_{\mathrm{p}}=0,09562+0,85534 * \mathrm{DB}_{1 \mathrm{~m}}$ & 0,91 & 1,84 \\
& $\mathrm{DB}_{\mathrm{p}}=0,23107+0,61005 * \mathrm{DB}_{\text {dap }}$ & 0,65 & 3,58 \\
Densidade Básica & $\mathrm{DB}_{\mathrm{p}}=0,03356+0,94299 * \mathrm{DB}_{2 \mathrm{~m}}$ & 0,91 & 1,85 \\
da madeira $\left(\mathrm{g} / \mathrm{cm}^{3}\right)$ & $\mathrm{DB}_{\mathrm{p}}=0,07089+0,86828 * \mathrm{DB}_{3 \mathrm{~m}}$ & 0,83 & 2,50 \\
& $\mathrm{DB}_{\mathrm{p}}=0,05304+0,88961 * \mathrm{DB}_{4 \mathrm{~m}}$ & 0,85 & 2,31 \\
& $\mathrm{DB}_{\mathrm{p}}=0,02322+0,94078 * \mathrm{DB}_{5 \mathrm{~m}}$ & 0,89 & 1,99 \\
& $\mathrm{DB}_{\mathrm{p}}=0,01813+0,95158^{*} \mathrm{DB}_{6 \mathrm{~m}}$ & 0,90 & 1,96 \\
& $\mathrm{DB}_{\mathrm{p}}=0,2361+0,42465 * \mathrm{DB}_{1 \mathrm{~m}}-$ & 0,97 & 0,92 \\
& $0,0600 / \mathrm{DB}_{3 \mathrm{~m}}+0,3658^{*} \mathrm{DB}_{6 \mathrm{~m}}$ & & \\
\hline
\end{tabular}

A baixa correlação apresentada entre a densidade ao nível do DAP e a densidade ponderada da árvore foi também observada por Campos, Silva e Vital (1992), ao estudar eucalipto de grande porte.

Já Valeri, Aguiar e Banzato (1990), trabalhando com eucalipto, e Vale, Martins e Araújo (1992), com duas espécies do cerrado (Vochysia thirsoidea Pohl e Pterodon pubescens Benth), encontraram que a densidade básica no DAP estima bem a densidade básica da árvore, sendo desta maneira representativa para converter volume em peso seco.

Uma opção que propiciou estimativas mais eficientes da densidade básica da árvore foi quando coletou-se amostra a 1,0; 3,0 e 6,0 m de altura da árvore. Neste caso o coeficiente de determinação $\left(\mathrm{R}^{2}\right)$ igual 0,97 e o erro padrão da estimativa igual $0,92 \%$. A distribuição de resíduo apresentada na Figura 5 apresentou tendência de superestimativa. No entanto, a amplitude de resíduos de $\pm 4 \%$ credencia o uso desta equação para estimativa do peso seco. Este resultado concorda com Campos, Silva e Vital (1992), que verificaram que a densidade básica da madeira estimada a partir de amostras retiradas ao longo do fuste é mais eficiente para estimar peso seco. 

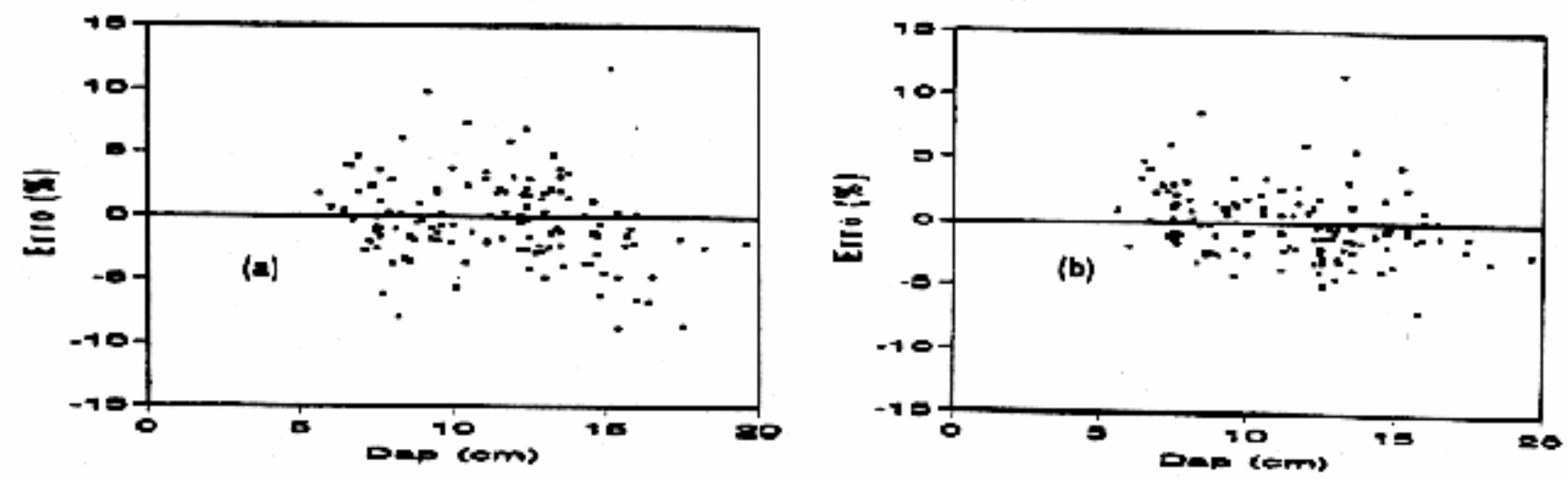

FIGURA 4: Distribuição gráfica dos resíduos para equações de densidade básica, para identificar a posição de coleta na árvore, para $1 \mathrm{~m}$ (a), para o DAP (b).

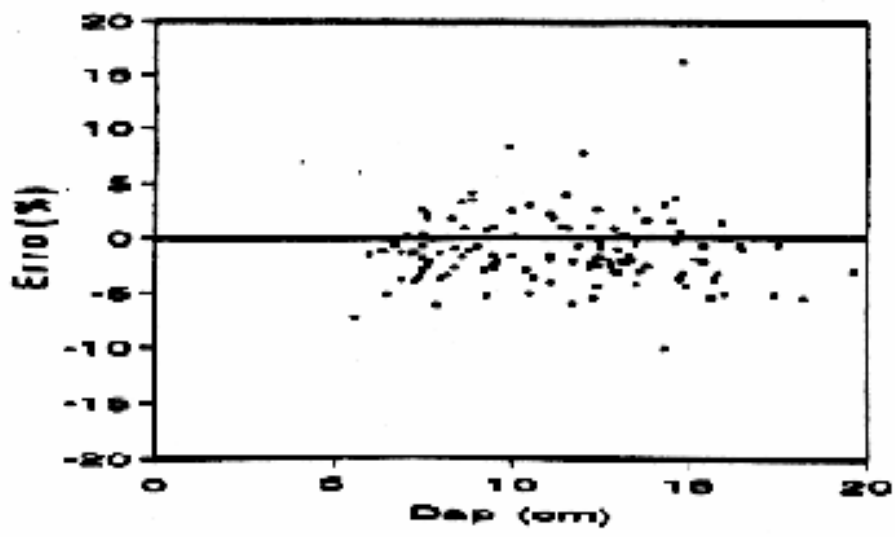

FIGURA 5: Distribuição gráfica dos resíduos para equações de densidade básica, para identificar a posição de coleta na árvore, para as posições de 1,3 e 6 metros.

Nenhuma abordagem da definição do ponto ótimo de amostragem da densidade básica na árvore em relação a idade foi mostrada, já que nenhum dos ajustes foi satisfatório. No entanto, constatou-se uma tendência de aumento nesta com acréscimo da idade. Esta relação também foi observada por Ferreira, Freita e Ferreira (1979), Jesus e Ferreira (1986) e Trugilho, Lima e Mendes (1996), entre outros.

\section{CONCLUSÃO}

A biomassa total com e sem casca pode ser estimada pela equação de simples entrada, dupla entrada, pela equação de dupla entrada associada à relação hipsométrica e pelo método dos dois diâmetros.

Ciência Florestal, v.8, n.1, 1998 
As medidas de precisão das equações que estimam o peso seco total com casca indicam estimativas mais confiáveis desta variável que do peso seco total sem casca.

A estimativa da biomassa pode ser obtida a custos mais baixos e com mesma eficiência que pelos métodos baseados em diâmetro e altura, quando faz-se uso do método dos dois diâmetros e da relação hipsométrica, associada à equação de dupla entrada.

Se for desejado obter a densidade básica da árvore a partir de uma amostra, esta deve ser tomada a 1,0 metro de altura na árvore, para Eucalyptus camaldulensis.

Se maior precisão for desejada na estimativa da densidade básica da árvore então amostra a 1,0; 3,0 e 6,0 metros na árvore devem ser tomadas.

\section{REFERÊNCIAS BIBLIOGRÁFICAS}

BRASIL, M.A.M.; VEIGA, R.A. de A.; TIMONI, J.L. Erros na determinação da densidade básica da madeira. Cerne. Lavras, v. 1, n. 1, p. 55-57, 1994.

BARRICHELO, L.E.G.; BRITO, J.O.; MIGLIORINI, A.J. Estudo da variação longitudinal da densidade básica de Eucalyptus spp. In: CONGRESSO FLORESTAL BRASILEIRO, 4, Belo Horizonte, 1982. Anais... Belo Horizonte: SBS, 1982. P 726 - 731.

CAMPOS, J.C.C.; SILVA, J.A. da; VITAL, B.R. Volume e biomassa do tronco e da copa de eucalipto de grande porte. Revista Árvore, Viçosa, v.16, n.3, p.319-336, set - dez 1992.

CAMPOS, J.C.C.; LEITE, H.G.; SOUZA, R.N. de; VITAL, B.R. Relações entre espaçamento, volume e peso de madeira em plantações de eucalipto. Revista Árvore. Viçosa, v. 14, n. 2, p. 119-133, jul - dez 1990.

FERREIRA, C.A.; FREITA, M. de; FERREIRA, M. Densidade básica da madeira de plantações comerciais de eucaliptos, na região de Mogi-Guaçu, São Paulo. IPEF, Piracicaba, n. 18, p.106117, jun 1979.

FINKE HERRERA, M.E. Densidade básica e equações de peso de madeira seca de povoamentos de eucaliptos de acordo com a idade, local, espécie e método de regeneração. Viçosa, UFV, 1989. 113p. (Dissertação de Mestrado).

FOELKEL, C.; MORA, E.; MENOCHELLI, S. Densidade básica: Sua verdadeira utilidade como índice de qualidade da madeira de eucalipto para produção de celulose. In: CONGRESSO FLORESTAL BRASILEIRO, 6, Campos do Jordão, 1990. Anais... : Campos do Jordão: SBSSBEF, 1990. p.719-728.

FRANCO, E.J. Estudo dos métodos estimativos de volume, biomassa e níveis de produtividade para Eucalyptus camaldulensis. Lavras. UFLA. 1996. 100p. (Dissertação de Mestrado)

GOMES F.P. Curso de Estatística Experimental. 13ª̣ ed, Piracicaba, Nobel, 1984. 468p.

JESUS, R.M.; FERREIRA, M. Comparação entre densidade de matrizes e de clones de 
Eucalyptus grandis. Revista Árvore, Viçosa, v.10, n.1, p.82-90, jan - jun 1986.

OLIVEIRA, A.D. de; LIMA, J.T.; ROSADO, S.C. da S. Estimativa da massa lenhosa em povoamentos de Eucalyptus spp. Ciência e Prática. Lavras, v.15, n. 4, p. 345-348, out - dez. 1991.

PANSHIN, A.J.; ZEUW, C. Textbook of wood technoloy. New York, McGraw-Hill, 1970, v. 1, $705 \mathrm{p}$.

SCOLFORO, J.R.S.; LIMA, J.T.; SILVA, S.T. Equações de biomassa e volume para cerrado senso stricto. In: CONGRESSO FLORESTAL PANAMERICANO, 1, e CONGRESSO FLORESTAL BRASILEIRO,7, Curitiba, 1993. Anais... : Curitiba: SBS-SBEF, 1993. p. 508-510.

SCOLFORO, J.R.S. Mensuração florestal 2: Volumetria. Lavras: ESAL/FAEPE, 126p. 1993.

SCOTT, A.J., KNOTT, M. A cluster analysis method for grouping means in the analysis of variance. Biometrics, v.30, p. 505-512, sept. 1974.

SHIMOYAMA, V.R. de S. Variação da densidade básica e características anatômicas e químicas da madeira em Eucalyptus spp. Piracicaba, ESALQ, 1990. 93p. (Dissertação de Mestrado).

SILVA, J.A. de A. Estimativas de volume do tronco e da copa, densidade básica, fator de empilhamento de madeira serrada de eucalipto. Viçosa, UFV, 1991. 93p. (Dissertação de Mestrado).

TRUGILHO, P.F.; SILVA, D.A. da; FRAZÃO, F.J.L.; REGAZZI, A.F. Caracterização de espécies nativas e exóticas amazônicas e do carvão vegetal. Revista Árvore, Viçosa, v.15, n.2, p.144151, mai - ago 1991.

TRUGILHO, P.F.; LIMA, J.T.; MENDES, L.M. Influência da idade nas características físicoquímicas e anatômicas da madeira de Eucalyptus saligna. Cerne. Lavras, v. 2, n.1, p. 94-116, 1996.

VALE, T. do; MARTINS, I.S.; ARAÚJO, W.L. de O. Estudo da densidade de três espécies do cerrado. Revista Árvore, Viçosa, v.16, n.2, p.209-217, mai - ago 1992.

VALERI, S.V.; AGUIAR, I.B.; BANZATTO, D.A. Variação da densidade básica da madeira de Eucalyptus grandis Hill ex-Maiden com a altura do caule e aplicação de fósforo e calcário dolomítico. In: CONGRESSO FLORESTAL BRASILEIRO, 6,Campos do Jordão, 1990. Anais... Campos do Jordão: SBS, 1990.

VITAL, B.R. Método de determinação da densidade da madeira. Viçosa: SIF, 1984. (Boletim Técnico 1). 